\title{
Extra-Parenchymal Chest HRCT Findings in Patients with Systemic Sclerosis at the Time of Initial Diagnosis
}

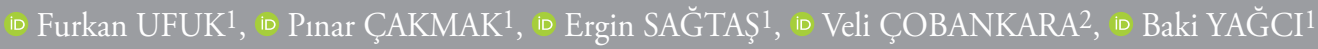

1 Pamukkale University Faculty of Medicine, Department of Radiology, Denizli, Turkey

2Pamukkale University Faculty of Medicine, Department of Rheumatology, Denizli, Turkey

\begin{abstract}
Objective: In systemic sclerosis (SS) patients who are receiving treatment, findings of extra-parenchymal chest involvement (such as thymic abnormality, mediastinal lymph node enlargement) have been previously examined. However, these findings may be affected by medical treatment. Our aim was to evaluate the extra-parenchymal chest high-resolution computed tomography (HRCT) findings of patients with SS at the time of initial diagnosis.

Methods: We retrospectively analyzed medical database of patients with SS. Chest HRCT images within 1 month after initial diagnosis of these patients were re-evaluated for the presence of distal esophageal dilatation, thymic hyperplasia, mediastinal lymph node enlargement, pleural or pericardial abnormalities (effusion or thickening). Intergroup comparisons were performed using independent t-test or a Mann-Whitney $\mathrm{U}$ test. To detect the relationship between continuous variables, Spearman's correlation coefficients and univariate correlations were used.

Results: A total of 51 patients ( 45 women and 6 men, mean age + STD; 49.2 years+13.9) with SS were included in the study. Esophageal dilatation (88.2\%) was the most common finding. Six patients (11.8\%) had thymic enlargement. Mediastinal lymph node enlargement (11.8\%) and pleural abnormalities $(11.8 \%)$ were significantly more common in patients with SS diagnosed at late age $(\mathrm{p}<0.05)$.

Conclusion: The extra-parenchymal findings are common in patients with SS at the time of initial diagnosis. Esophageal dilatation is usually present at the time of diagnosis in most patients with SS regardless of age.
\end{abstract}

Keywords: Systemic sclerosis, scleroderma, high-resolution computed tomography, thymus, lymph node

\section{Introduction}

Systemic sclerosis (SS), also known as scleroderma, is an autoimmune collagen vascular disease and it causes abnormal collagen growth in tissues such as skin, lung, heart and digestive tract. SS is usually characterized by swelling in the fingertips, joint pain and excessive tension and hardness of skin (1). Chest highresolution computed tomography (HRCT) significantly increases the diagnostic sensitivity of lung involvement in SS (1-4). Also, mediastinal lymph node enlargement (MLNE), pleural disease or thymic enlargement may be seen in patients with SS at any time during the illness (5-10). However, the extra-parenchymal findings of SS may change with medical treatment. For example, thymus gland size decreases in response to use of corticosteroids or immunosuppressants. However, the risk of MLNE and pleural disease (pleural effusion or thickening) increases because of the increased frequency of infection in patients using corticosteroids or immunosuppressants $(11,12)$. To the best of our knowledge, there is no study that evaluates extra-parenchymal chest CT findings in newly diagnosed patients with SS. Therefore, our aim was to evaluate the extra-parenchymal chest HRCT findings in newly diagnosed patients with SS and compare with literature.

Address for Correspondence: Furkan UFUK, Pamukkale University Faculty of Medicine, Department of Radiology, Denizli, Turkey

Phone: +90 +90 5545115088 E-mail: furkan.ufuk@hotmail.com ORCID ID: orcid.org/0000-0002-8614-538

Cite this article as: Ufuk F, Çakmak P, Sağtaş E, Çobankara V, Yağcı B. Extra-Parenchymal Chest HRCT Findings in Patients with Systemic Sclerosis at the Time of Initial Diagnosis. Bezmialem Science 2019;7(2):101-6. 
We also aimed to demonstrate the relationship between the age at initial diagnosis and extra-parenchymal HRCT findings.

\section{Methods}

This single-center retrospective study was approved by our institutional local ethics committee. This single-center retrospective study received approval from Pamukkale University, Medical Ethics Committee (03.10.2017/13).

\section{Patients}

The patients diagnosed as having SS according to the criteria of the American Rheumatism Association 1980 (13) or ACR/ EULAR 2013 classification criteria (14) for SS, between January 2009 and August 2017 were retrospectively analyzed.

The patients with SS who underwent HRCT scan within one month after the initial diagnosis were included in the study. Having respiratory symptoms was indication for performing HRCT. Patients with history of malignancy, esophageal-gastric surgery or smoking and patients with additional rheumatologic disease (such as rheumatoid arthritis, Sjögren syndrome) or patients with overlap syndromes (such as SS and Sjögren syndrome overlap syndrome) were excluded from the study. Patients without HRCT screening within one month after initial diagnosis were not included in the study.

\section{High-Resolution Computed Tomography (HRCT) Scanning Protocol and Radiological Evaluation}

All HRCT scans were obtained in the supine position with using a multi-detector CT system (Brilliance 16; Philips Healthcare, Best, the Netherlands) with full inspiration and the following parameters: $\mathrm{Kv}, 140$; mAs, 280; detector collimation, 16x0.8/0.4 $\mathrm{mm}$; slice thickness, $1 \mathrm{~mm}$; slice interval, $10 \mathrm{~mm}$; field of view (FOV), $35 \mathrm{~cm}$ and matrix, $512 \times 512$. Expiratory scans at least three selected levels (aortic arch, $2 \mathrm{~cm}$ above the diaphragm and tracheal carina) were obtained.

All HRCT images of the patients with SS were evaluated by two radiologists by consensus. Axial HRCT images of the chest were evaluated in the lung window for the presence of distal esophageal dilatation (with a maximum esophageal diameter $\geq 9 \mathrm{~mm}$ ). Also HRCT images were evaluated in the mediastinal window for the presence of thymic hyperplasia (TH) (increased thymic thickness with $\geq 13 \mathrm{~mm}$ ), MLNE (MLNE) (increased short-axis diameter with $\geq 10 \mathrm{~mm}$ ), pleural or pericardial abnormalities (effusion or thickening).

\section{Statistical Analysis}

Statistical analysis was performed using the Statistical Package for the Social Sciences (SPSS) for Windows (Version 19.0: SPSS Inc. IBM Corp, Chicago, IL, USA). Continuous and categorical data were reported as mean \pm standart deviation (SD), frequency and percentage. Intergroup comparisons were performed using independent t-test or a Mann-Whitney $\mathrm{U}$ test for continuous variables. Spearman's correlation coefficients and univariate correlations were used to detect the relationship between continuous variables. $\mathrm{P}<0.05$ was considered to indicate statistical significance.

\section{Results}

Medical database analysis revealed a total of 101 patients who were newly diagnosed as having SS between January 2009 and August 2017. Among these patients, 4 patients with history of malignancy ( 2 breast, 1 lung, 1 rectum cancer), 9 patients with additional rheumatic disease or overlap syndromes (4 Sjögren syndrome, 2 rheumatoid arthritis, 2 dermatomyositis, 1 systemic lupus erythematosus) and 9 patients with smoking history were excluded from the study. In addition, 26 patients with $>1$-month interval between imaging and initial diagnosis, 2 patients with severe artifacts on HRCT. Totally, 51 patients [88.2\% $(\mathrm{n}=45)$ females, $11.8 \%(\mathrm{n}=6)$ males]; whose mean age \pm STD was 49.2 years \pm 13.9 (range 18-71 years) and who met the appropriate conditions were included in the study. The median time between SS diagnosis and HRCT examination was 8 days (range: 1-23 days).

The HRCT findings (thymic enlargement, MLNE, esophageal dilatation, pleural-pericardial abnormalities) and frequencies are shown in Table 1 and 2. Also, correlations of variables are shown in Table 3. The HRCT images of the patients are shown in Figures 1-4.

\section{Table 1. High-resolution computed tomography findings in patients with systemic sclerosis}

\begin{tabular}{|l|l|l}
\hline HRCT Finding & Yes & No \\
\hline Thymic enlargement & $n(\%)$ & $n(\%)$ \\
\hline MLNE & $6(11.8 \%)$ & $45(88.2 \%)$ \\
\hline Esophagus dilatation & $6(11.8 \%)$ & $45(88.2 \%)$ \\
\hline Pleural abnormalities & $45(88.2 \%)$ & $6(11.8 \%)$ \\
\hline Pericardial abnormalities & $6(11.8 \%)$ & $45(88.2 \%)$ \\
\hline
\end{tabular}

HRCT: High-resolution computed tomography, MLNE: Mediastinal lymph node enlargement, n: Number of patients 
Four patients had pericardial effusion (7.8\%). Pericardial thickening was not seen in any patient. Six patients $(11.8 \%)$ had pleural abnormality, including effusion $(n=4)$, and focal pleural thickening $(n=2)$.
There was no significant difference between male and female patients in terms of thymic enlargement ( $\mathrm{p}=0.699)$, MLNE $(\mathrm{p}=0.351)$, esophagus dilatation $(\mathrm{p}=0.699)$, pleural $(\mathrm{p}=0.699)$ and pericardial abnormalities $(\mathrm{p}=0.457)$.

Table 2. Comparison of high-resolution computed tomography findings in patients with systemic sclerosis with mean age of the patients

\begin{tabular}{l|l|l|l}
\hline HRCT Finding & Yes & No & p value \\
& Mean Age & Mean Age & 0.441 \\
\hline Thymic enlargement & 44 years & 49.9 years & 0.021 \\
MLNE & 61.3 years & 48.6 years & 0.166 \\
Esophagus dilatation & 50.2 years & 41.8 years & 0.002 \\
Pleural abnormalities & 64.8 years & 47.1 years & 0.054 \\
\hline Pericardial abnormalities & 62 years & 48.1 years & \\
\hline HRCT: High-resolution computed tomography, MLNE: Mediastinal lymph node enlargement, n: Number of patients, p<0.05 means statistical significance
\end{tabular}

Table 3. Correlations of variables

\begin{tabular}{|c|c|c|c|c|c|}
\hline HRCT Finding & 1 & 2 & 3 & 4 & 5 \\
\hline 3. Esophageal dilatation & 0.13 & 0.13 & 1 & 0.13 & 0.11 \\
\hline 4. Pleural abnormality & -0.13 & $0.62 * *$ & 0.13 & 1 & $0.35^{*}$ \\
\hline
\end{tabular}

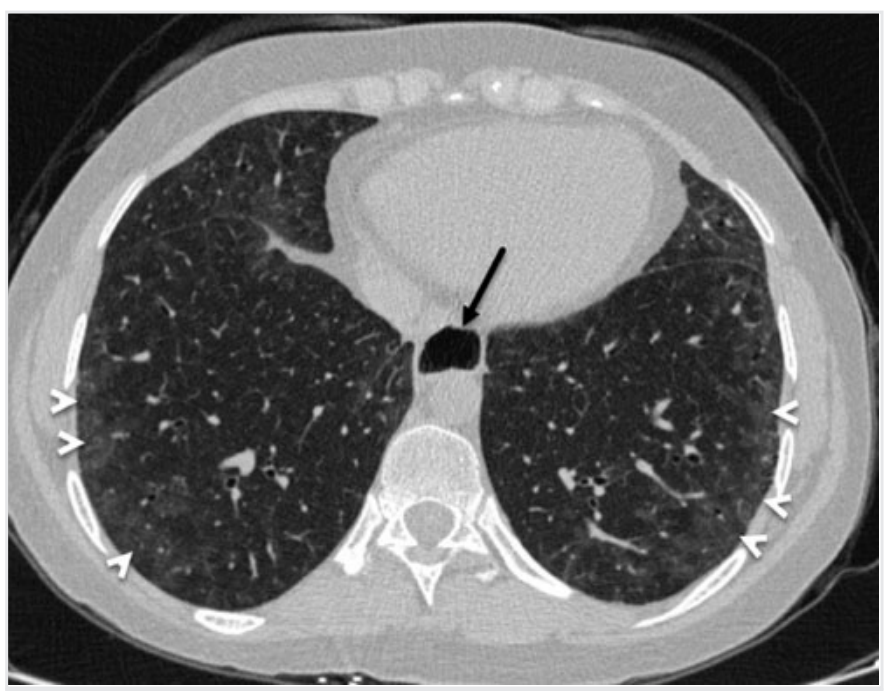

Figure 1. A 42-year-old female with systemic sclerosis. Axial high-resolution computed tomography image obtained during deep inspiration shows ground-glass opacity areas (arrow heads). Also, distal esophageal dilatation is seen (black arrow)

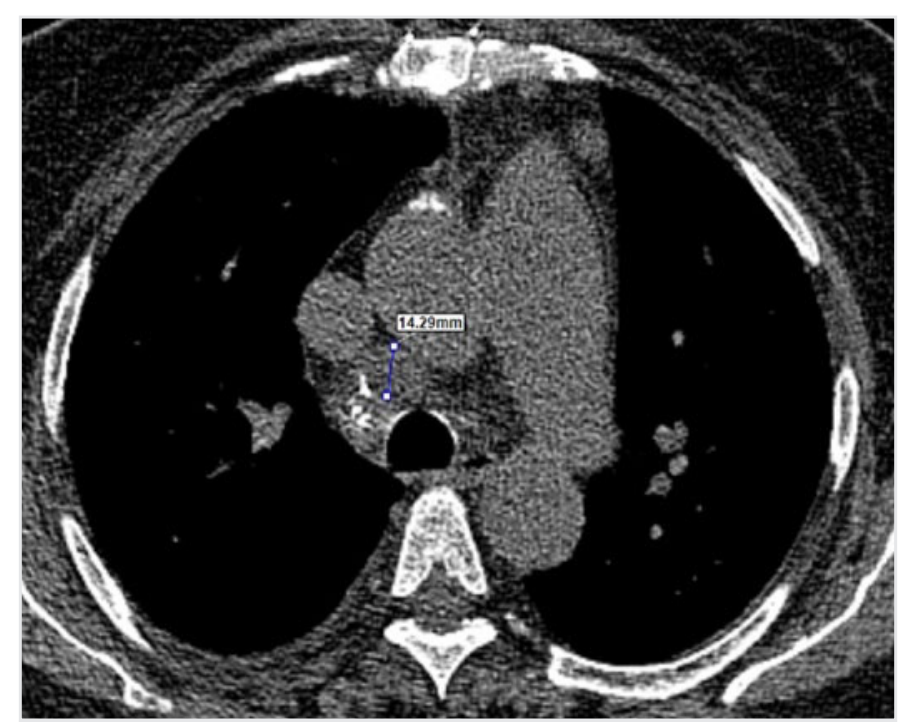

Figure 2. A 56-year-old female with systemic sclerosis. Axial high-resolution computed tomography image shows right para-tracheal lymph node enlargement with a $14 \mathrm{~mm}$ short axis 


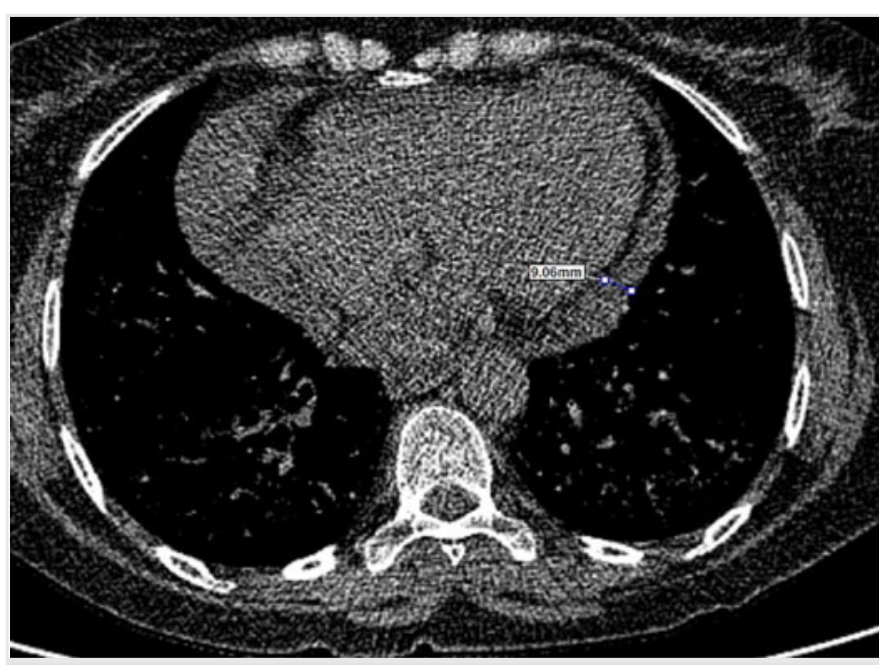

Figure 3. A 48-year-old female with systemic sclerosis. Axial high-resolution computed tomography image obtained during deep inspiration shows pericardial effusion with a 9 $\mathrm{mm}$ thickness

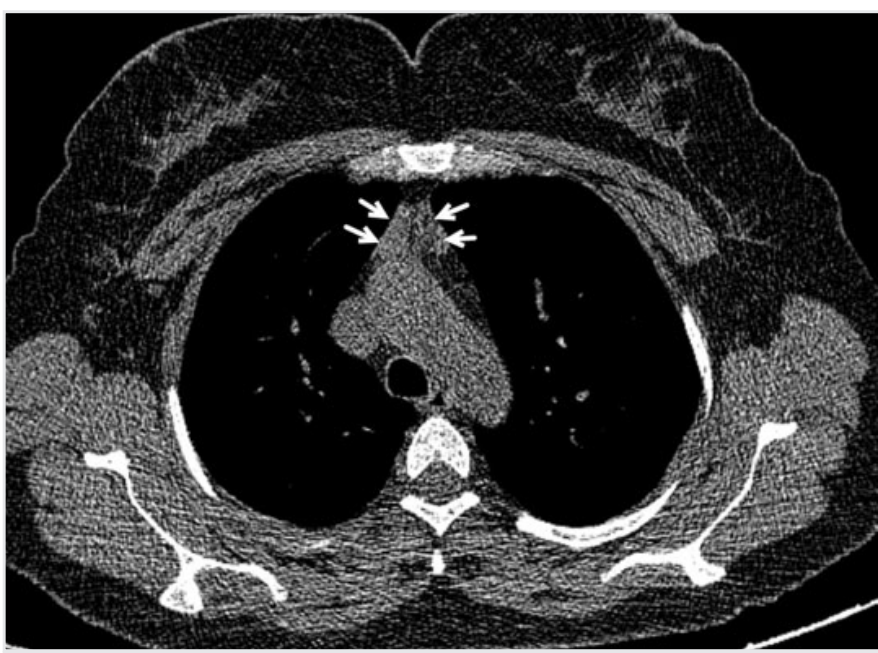

Figure 4. A 36-year-old male with systemic sclerosis. Axial high-resolution computed tomography image shows thymic enlargement with a $21 \mathrm{~mm}$ thickness (white arrows)

\section{Discussion}

According to our results, esophageal dilatation (88.2\%) is frequently found in SS patients with respiratory symptoms, at the time of initial diagnosis. MLNE and pleural abnormalities are significantly more common in patients with SS diagnosis at the late age.

Although the actual role of thymic abnormalities on imaging studies in patients with SS is still unclear, the possible involvement of the immune-mediated SS pathogenesis at an early stage may be presumed $(7,8,14)$. When the increased thymic thickness was accepted as a threshold value of greater than $13 \mathrm{~mm}$, Colaci et al. (15) reported that the frequency of abnormalities in thymus size was $9.5 \%$ (19 of 200 patients) in patients with SS with a mean of 5-year (0-38 years) disease duration. Ferri et al. (7) found that, radiological thymic enlargement were correlated with SS disease duration and thymic enlargement more common in patients with short disease duration $(\leq 5$ years or $>5$ years; $\mathrm{p}=0.007)$. They detected thymic enlargement in 9 of 34 cases (26\%). We found that thymic enlargement was present in $11.8 \%$ (6 of 51) of patients with SS, at the time of initial diagnosis. In addition, studies showed that patients with SS with thymic enlargement were found at a younger age. For this reason, it has been argued that thymic enlargement is due to insufficient involution of the thymus gland (16-18). In our study, although the mean age of cases with thymic enlargement was lower than that of without thymic enlargement, there was no statistically significant difference between the age of initial diagnosis and presence of thymic enlargement (44 vs 49.9 years; $\mathrm{p}=0.441$ ). This may be due to a small number of cases in our study. In our study, unlike previous studies, the HRCT findings of newly diagnosed patients with SS were evaluated. We suggest that the duration of the disease and the medications may cause thymic abnormalities. There is a need for more extensive studies in order to explain this situation.

MLNE has been reported to be a common finding in patients with SS (9). In patients with SS, Santos et al. (5) demonstrated that, MLNE was present in 5 of 23 patients (21\%), Yamakawa et al. (17) found that MLNE was seen in $20 \%(\mathrm{n}=40)$, and Farrokh et al. (18) found that $30 \%(\mathrm{n}=40)$ of patients with SS had MLNE on HRCT. Wechsler et al. (19) and Garber et al. (20) found that MLNE was present in $\% 50$ and 32\% of patients with SS, respectively. In those studies, examining the prevalence of MLNE, the duration of the disease and medical treatment were not considered (5,9,17-20). In our study, MLNE was present in $11.8 \%$ of newly diagnosed SS patients $(n=51)$. The mean age of the patients with MLNE was significantly higher than those without MLNE ( $\mathrm{p}=0.021)$. It is more likely that elderly patients have more SS disease duration and risk of infectious lung diseases than younger ones.

Another common finding in patients with SS is esophageal involvement. Esophageal involvement is common in SS and it can occur from 58 to $80 \%$ in different studies $(9,21,22)$. Vonk et al. (21) reported that, esophageal dilation in patients with interstitial lung disease suggests a diagnosis of SS with a high sensitivity and specificity on HRCT ( $63 \%$ and $88 \%$, respectively). In our study, we demonstrated that esophageal dilation is a frequent HRCT finding in patients with SS at the time of initial diagnosis, with a rate of $88.2 \%(45 / 51)$.

It has been reported that pleural abnormalities are rarely present in patients with SS. Pleural abnormalities may be seen as, diffuse or focal pleural thickening, pleural effusion, and pseudo-plaques (22). Farrokh et al. (18) reported that pleural abnormality was present in $30 \%$ of patients (12 of 40) with SS and they found 4 patients had pleural effusion, 8 patients had pleural thickening. Yamakawa et al. (17) found that, pleural abnormality (thickening or effusion) was present in $10 \%$ of patients with SS $(n=40)$. Pleural effusion in patients with SS is an uncommon finding that requires a complete evaluation to exclude different causes of pleural effusion such as heart failure, side effects of medication and pneumonia (23). Similar to Yamakawa et al. (17), we also 
detected that $11.8 \%$ of SS patients had pleural abnormalities on HRCT. We also found that, as expected, the age of the patients with pleural abnormality was significantly higher than the age of the patients without pleural abnormality $(p=0.002)$. This may be due to the fact that elderly patients are more likely to develop empyema or hemothorax than younger patients. Also, pleural abnormality may occur due to long disease duration or heart failure in the elderly.

This study has some limitations. First, the HRCT images were evaluated by two observers by consensus so the differences between the observers could not be assessed. Second, it was a retrospective study that involved a relatively small number of cases. Third, there was also sampling bias since only patients with positive respiratory symptoms were included in the study. So, we could not evaluate the extra-parenchymal chest $\mathrm{CT}$ findings in patients without respiratory symptoms. Finally, we did not have histopathological findings and we could not make a radiologicalhistopathological comparison.

\section{Conclusion}

Despite these limitations, our study suggests that esophageal dilatation is frequently found in patients with SS with respiratory symptoms at the time of initial diagnosis. MLNE and pleural effusion are more common in newly diagnosed patients with SS at the late age, probably due to long disease duration and advanced age.

\section{Ethics}

Ethics Committee Approval: This single-center retrospective study received approval from Pamukkale University, Medical Ethics Committee (03.10.2017/13).

Informed Consent: Written informed consent was not obtained from participants due to retrospective nature of study.

Peer review: Externally peer-reviewed.

\section{Authorship Contributions}

Concept: F.U., P.Ç., Design: F.U., E.S., Data Collection or Processing: F.U., Analysis or Interpretation: F.U., Literature Search: E.S., P.Ç., Writing: F.U., B.Y.

Conflict of Interest: No conflict of interest was declared by the authors.

Financial Disclosure: The authors declared that this study has received no financial support.

\section{References}

1. Fischer A, West SG, Swigris JJ, Brown KK, du Bois RM. Connective tissue disease-associated interstitial lung disease: a call for clarification. Chest 2010;138:251-6.

2. Solomon JJ, Olson AL, Fischer A, Bull T, Brown KK, Raghu G. Scleroderma lung disease. Eur Respir Rev 2013;22:6-19.

3. Ioannidis JP, Vlachoyiannopoulos PG, Haidich AB et al. Mortality in systemic sclerosis: an international meta-analysis of individual patient data. Am J Med 2005;118:2-10.
4. Lynch DA. Lung disease related to collagen vascular disease. J Thorac Imaging 2009;24:299-309.

5. Santos MK, Faria FB, Trad CS. Pulmonary involvement in systemic sclerosis: cases review. Radiol Bras 2006;39:181-4.

6. Denton CP, Khanna D. Systemic sclerosis. Lancet 2017;390:168599.

7. Ferri C, Colaci M, Battolla L, Giuggioli D, Sebastiani M. Thymus alterations and systemic sclerosis. Rheumatology (Oxford) 2006;45:72-5.

8. Oksel F, Tarhan F, Bayraktaroğlu S, Savaş R, Yargucu F, Keser G. Radiological incomplete thymus involution in systemic sclerosis. Rheumatology 2009;48:800-3.

9. Bhalla M, Silver RM, Shepard JA, McLoud TC. Chest CT in patients with scleroderma: prevalence of asymptomatic esophageal dilatation and mediastinal lymphadenopathy. AJR Am J Roentgenol 1993;161:269-72.

10. Highland KB, Heffner JE. Pleural effusion in interstitial lung disease. Curr Opin Pulm Med 2004;10:390-6.

11. Webb RW. The mediastinum: mediastinal masses. Thoracic imaging: pulmonary and cardiovascular radiology. In: Webb RW, Higgins C, editors. Philadelphia: Lippincott Williams \& Wilkins; 2005:212-70.

12. Kanne JP, Yandow DR, Meyer CA. Pneumocystis jiroveci Pneumonia: High-Resolution CT Findings in Patients With and Without HIV Infection. AJR Am J Roentgenol 2012;198:W555-61.

13. Preliminary criteria for the classification of systemic sclerosis (scleroderma). Subcommittee for scleroderma criteria of the American Rheumatism Association Diagnostic and Therapeutic Criteria Committee. Arthritis Rheum 1980;23:581-90.

14. van den Hoogen F, Khanna D, Fransen J, Johnson SR, Baron M, Tyndall A, et al. 2013 classification criteria for systemic sclerosis: an American college of rheumatology/European league against rheumatism collaborative initiative. Ann Rheum Dis 2013;72:174755.

15. Colaci M, Giuggioli D, Manfredi A, Vacchi C, Della Casa G, Ferri C. Radiological thymus alterations in systemic sclerosis: our experience and a review of the literature. Rheumatology (Oxford) 2014;53:7326.

16. Meunier M, Bazeli R, Feydy A, Drape JL, Kahan A, Allanore Y. Incomplete thymic involution in systemic sclerosis and rheumatoid arthritis. Joint Bone Spine 2013;80:48-51.

17. Yamakawa H, Hagiwara E, Kitamura H, Yamanaka Y, Ikeda S, Sekine A, et al. Clinical Features of Idiopathic Interstitial Pneumonia with Systemic Sclerosis-Related Autoantibody in Comparison with Interstitial Pneumonia with Systemic Sclerosis. PLoS One 2016;11:e0161908.

18. Farrokh D, Abbasi B, Fallah-Rastegar Y, Mirfeizi Z. The Extrapulmonary Manifestations of Systemic Sclerosis on Chest High Resolution Computed Tomography. Tanaffos 2015;14:193-200.

19. Wechsler RJ, Steiner RM, Spirn PW, Rubin S, Salazar AM, Shah R, et al. The relationship of thoracic lymphadenopathy to pulmonary interstitial disease in diffuse and limited systemic sclerosis: CT findings. AJR Am J Roentgenol 1996;167:101-4. 
20. Garber SJ, Wells AU, duBois RM, Hansell DM. Enlarged mediastinal lymph nodes in the fibrosing alveolitis of systemic sclerosis. Br J Radiol 1992;65:983-6.

21. Vonk MC, Van Die CE, Snoeren MM, Bhansing KJ, Van Riel PL, Fransen J, et al. Oesophageal dilatation on high-resolution computed tomography scan of the lungs as a sign of Scleroderma. Ann Rheum Dis 2008;67:1317-21.
22. Pandey AK, Wilcox P, Mayo JR, Moss R, Ellis J, Brown J, et al. Oesophageal dilatation on high-resolution CT chest in systemic sclerosis: What does it signify? J Med Imaging Radiat Oncol 2011;55:535-641.

23. Highland KB, Heffner JE. Pleural effusion in interstitial lung disease. Curr Opin Pulm Med 2004;10:390-6. 